Volume 8, No.1.5, 2019

International Journal of Advanced Trends in Computer Science and Engineering

Available Online at http://www.warse.org/IJATCSE/static/pdf/file/ijatcse0881.52019.pdf

https://doi.org/10.30534/ijatcse/2019/0881.52019

\title{
Bezier and Trigonometric Bezier with a Shape Parameter Curve in Designing 3-Dimensional Objects Using Rotational Sweep Surface Method
}

\author{
Noor Khairiah Binti Razali ${ }^{1}$, Siti Musliha Binti Nor-Al-Din ${ }^{2}$, Nursyazni Binti Mohamad Sukri ${ }^{3}$, \\ Muhammad Amirul Bin Adenan ${ }^{4}$, Muhammad Hilmi Bin Abdul Azib ${ }^{5}$ \\ ${ }^{1}$ Universiti Teknologi MARA, Malaysia, noorkhairiah@uitm.edu.my \\ ${ }^{2}$ Universiti Teknologi MARA, Malaysia, sitim907@uitm.edu.my \\ ${ }^{3}$ Universiti Teknologi MARA, Malaysia, nursyazni@uitm.edu.my \\ ${ }^{4}$ Universiti Teknologi MARA, Malaysia, muhdmirul1195@gmail.com \\ ${ }^{5}$ Universiti Teknologi MARA, Malaysia, hilmiazib1996z@gmail.com
}

\begin{abstract}
Designing 3-dimensional objects are important in order to meet the customers' need. There are many objects that have been created and used in for various purposes such as products packaging, decorations and others. This paper focuses on designing 3-dimensional symmetrical objects which is a bottle design. The shape and configuration are significant for showcasing systems. The shape, size and shade of the 3-dimensional symmetrical objects are additionally influencing to advertising procedures. The design was produced by using combination methods of cubic Bezier and Trigonometry Bezier with a shape parameter with the Rotational Sweep Surface method. In this paper, the manipulation of the design was made based on the properties of both curves that is used to produce 2-dimensional designs. The designs were focused on the manipulation of control points and the value of shape parameter. After the manipulation stage, the 2-dimensional designs of two methods were converted into 3-dimensional design using the Rotational Sweep Surface method. After that, the best design was chosen after the manipulated stage. The best designs were chosen based on the simpler method in designing the variety shapes of bottle that can satisfy the consumers' needs. Based on the result, it is shown that Trigonometry Bezier with a shape parameter is the best method to be used than Cubic Bezier Curve.
\end{abstract}

Key words: Bezier Curve, 3-dimensional, Trigonometric Bezier, Sweep Surface.

\section{INTRODUCTION}

CAGD is an idea on the made of curve and surface displayed and also a new form in order to develop and make the focal points of improvement in industries, for example, car, aviation, and shipbuilding [6, 7]. In the year of 1971 in Paris CAGD focused on automotive design and was coined by P. Bezier [2]. This paper focuses on the designing of a bottle using the curve of cubic Bezier and Trigonometry with a shape parameter and the Rotational Sweep Surface method.
In designing bottle products, shapes are very important feature for marketing strategies. According to a study on the design of consumer packaging on, the effects of manipulations of shape orientation and alignment of graphical forms on consumer's assessments, the behavioral scientists found that rounded designs result in greater likelihood of purchase because they are more appealing and pleasing to the consumer. From another case study where the individuals responded to bottle packaging around two fundamental dimensions which are the first dimension, the customers' preferences are usually affected by the bottle shape, size, and colour and the second dimension is the dress of the bottle that can be considered as the basis of other packaging elements [13].

Bezier Curve was introduced by Pierre Etienne Bezier in the early 1960s. Bezier curve is a parametric bend $\mathrm{P}(\mathrm{t})$ that is a polynomial capacity of the parameter $t$. The level of the polynomial relies upon the quantity of control points used to characterize the bend [9]. The cubic Bezier curve used in this research has four control points of each curve.

Meanwhile, the cubic Trigonometric Bezier with a shape parameter has a section for each bend that will be created with four continuous control focuses and the state of the bend can be balanced by adjusting the estimation of shape parameters while the control of polygon will remain unaltered [11, 12]. This curve was an advanced curve that was added on with a shape parameter that made the curve easier to manipulate and was made different by changing the shape parameter's value [1].

The Sweep Surface is one of the 3-dimensional transformations that uses rotation, translation, scaling, reflection and shearing method. This method is a modeler for applications such as CAD/CAM and robotics that usually involves in sweeping bodies that does not change the shape because frames can be generated automatically by moving the body. This method is really useful for geometric design that we want to use for our research because it composes the surface of revolution and extruded surface [5].

This paper studies the properties of the curves in order to design bottle shape using cubic Bezier Curve and Cubic 
Trigonometry with a Shape Parameter with rotational sweep surface method. The curves were manipulated based on the control points and the value of shape parameter in order to generate the 3-dimensional designs. After manipulation, both methods were compared based on the shape of designs and then were focused on the easier method to manipulate and the variety designs produced.

\section{METHODOLOGY}

This study used two methods in generating 2-dimensional curves which are Cubic Bezier [8] and Cubic Trigonometric Bezier with a shape parameter [4]. Then, the Rotational sweep surface method was used in generating 3-dimensional objects. The objects chosen was a bottle design which is satisfied with the symmetrical rotational objects.

\subsection{Bezier Curve}

Pierre Etienne Bezier (1960) was a practical mathematician attached with a French car manufacturer Renault and was the creator of the Bezier Method. This method was implemented in process of designing a car [8].

Bezier curve is a parametric curve $\mathrm{P}(\mathrm{t})$ that is a polynomial capacity of the parameter $t$ [8]. The Bezier curve can be adjusted based on the control points and also the degree of the curve. The curve is close to the control polygon if the degree was increased. However, closer curve does not mean that smooth curve is produced. This paper used the cubic Bezier curve which is have four consecutive control points that is called as control polygon. This curve is commonly used in several applications as compare to the lowest and highest degree.

The equation cubic Bezier curves [8] is given in (1) and the basis graph and curve generated from (1) are shown in Figure 1.

$\mathrm{P}(\mathrm{t})=\sum_{\mathrm{t}=0}^{\mathrm{n}} \mathrm{P}_{\mathrm{t}} \mathrm{B}_{\mathrm{n}, \mathrm{i}}(\mathrm{t}), \quad 0 \leq \mathrm{t} \leq 1$

where

$$
B_{n, t}(t)=\left(\begin{array}{l}
n \\
t
\end{array}\right) t^{i}(1-t)^{n-i}
$$

and

$$
\left(\begin{array}{l}
\mathrm{n} \\
\mathrm{i}
\end{array}\right)=\frac{\mathrm{n} !}{\mathrm{i} !(\mathrm{n}-\mathrm{i}) !}
$$

$P(t)=(1-t)^{3} P_{0}+3(1-t)^{2} t P_{1}+3(1-t)^{2} P_{2}+t^{3} P_{3}$

where,

$$
\begin{aligned}
& P(t) \text { : Cubic Bezier curve } \\
& P_{n}: \text { Control Point } \\
& B_{i}(t): \text { Basis of Cubic Bezier }
\end{aligned}
$$
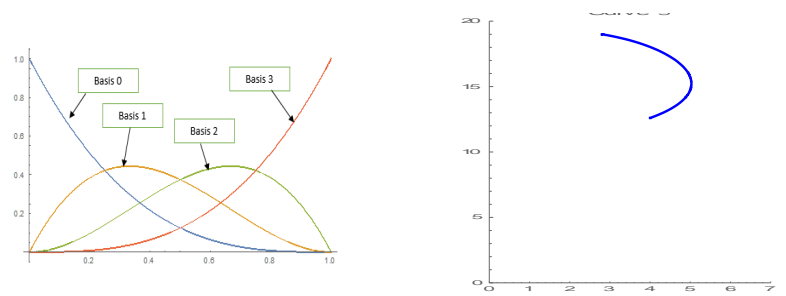

Figure 1: The basis graph and curve for Bezier function.

\subsection{Cubic Trigonometric Bezier with a Shape Parameter}

A cubic trigonometric Bezier curve is alike to the cubic Bezier curve, with a shape parameter, where the shape of the curve can be adjusted by changing the values while the control polygon is untouched. With shape parameters, the cubic trigonometric Bezier curves can be made closer to the cubic Bezier curves or closer to the given control polygon than the cubic Bezier curves [4].

The equation of cubic Trigonometric Bezier Curve with a shape parameter is defined by [4] and is given in (2). Then, the basis graph and curve for shape parameter $-2,-1$ and 1 are shown in Figures 2 and 3.

$$
r(t)=\sum_{i=0}^{3} P_{i} b_{i}(t), t \in[0,1] \text { and } \lambda=[-2,1]
$$

where,

$$
\begin{aligned}
& b_{i}(t)=\left\{\begin{array}{c}
b_{0}(t)=\left(1-\sin \frac{\pi}{2} t\right)^{2}\left(1-\lambda \sin \frac{\pi}{2} t\right) \\
b_{1}(t)=\sin \frac{\pi}{2} t\left(1-\sin \frac{\pi}{2} t\right)\left(2+\lambda-\lambda \sin \frac{\pi}{2} t\right) \\
b_{2}(t)=\cos \frac{\pi}{2} t\left(1-\cos \frac{\pi}{2} t\right)\left(2+\lambda-\lambda \cos \frac{\pi}{2} t\right) \\
b_{3}(t)=\left(1-\cos \frac{\pi}{2} t\right)^{2}\left(1-\lambda \cos \frac{\pi}{2} t\right)
\end{array}\right. \\
& t=\text { point on curve } \\
& \mathrm{B}_{\mathrm{n}, \mathrm{i}}=\text { Bernstein polynomial } \mathrm{s} \\
& \lambda=\text { parameter } \\
& \mathrm{P}=\text { control point }
\end{aligned}
$$

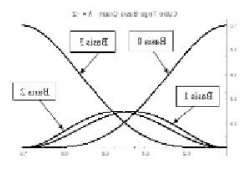

$\lambda=-2$

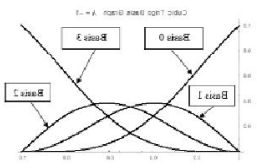

$\lambda=-1$

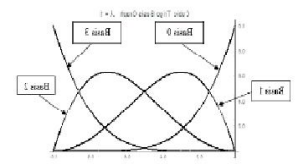

$\lambda=1$
Figure 2: The basis graph of Cubic Trigonometric Bezier 

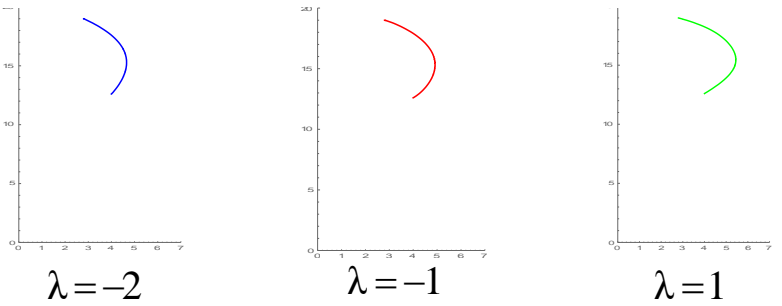

Figure 3: The curve of Cubic Trigonometric Bezier

\subsection{Sweep Surfaces Method}

This method is really useful for geometric design that we want to use for our research because it composes the surface of revolution and extruded surface. It is pragmatic, straightforward and effective to be utilized. Additionally, sweep surface called as summed up barrel and can be characterized as a wrapped frame. The curve and surface that are items in CAD and CAGD must be smooth and frame the exact shape [10].

A swept surface is produced by creating the curve and moving sideways with another curve, whereas optionally rotating and scaling, in order to make a surface patch. Rotation is one of technique that used in CAD/CAGD to produce the symmetric surface, whereby the two dimensional cross-section curves will be rotated at an axis which are can be parallel to the $\mathrm{x}$-axis, $\mathrm{y}$-axis and $\mathrm{z}$-axis. This method was simplest if the equation used in matrix operation [8]. Based on this method, if the angle of rotation is $\mathrm{w}$ and the curve will rotate at the axis $\mathrm{r}=\left(\mathrm{r}_{\mathrm{x}}, \mathrm{r}_{\mathrm{y}}, \mathrm{r}_{\mathrm{z}}\right)$, then the rotation matrix was simplified based on the axis are represent below:

$\begin{array}{rlrl} & & & \\ \text {-axis } & \mathrm{T}(\mathrm{w}) & =\left[\begin{array}{ccc}1 & 0 & 0 \\ 0 & \cos \mathrm{w} & \sin \mathrm{w} \\ 0 & -\sin \mathrm{w} & \cos \mathrm{w} 1\end{array}\right] \\ \text { y-axis } & \mathrm{T}(\mathrm{w})=\left[\begin{array}{ccc}\cos \mathrm{w} & 0 & \sin \mathrm{w} \\ 0 & 1 & 0 \\ -\sin \mathrm{w} & 0 & \cos \mathrm{w}\end{array}\right] \\ \text { z-axis } & \mathrm{T}(\mathrm{w})=\left[\begin{array}{cccc}\cos \mathrm{w} & \sin \mathrm{w} & 0 \\ -\sin \mathrm{w} & \cos \mathrm{w} & 0 \\ 0 & 0 & 1\end{array}\right]\end{array}$

The rotation surface is produced by using the equation (3):

$P(t, w)=P(t) \cdot T(w)$

where,

$$
\begin{aligned}
& P(t, w)=\text { Surface Revolution } \\
& P(t)=\text { Cross Section } \\
& T(w)=\text { Matrix Revolution }
\end{aligned}
$$

\section{DESIGNING 3-DIMENSIONAL OBJECTS}

The data was collected using the design of a reference 2-dimensional bottle design that has been drawn on the graph paper. Then, the control point was determined based on four consecutive points. From Figure 4, there 9 curves defined and from this control point, the 2-dimensional curves were generated using the cubic of Bezier curve and Trigonometric Bezier curves with a shape parameter. Then, the 2-dimensional designs were applied with the Rotational Sweep surface method to get the 3-dimensional design.

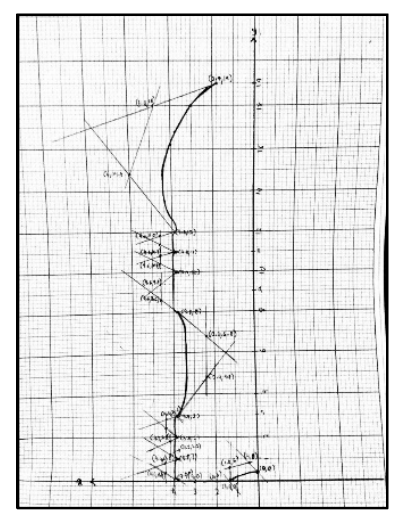

Figure 4: Reference Design

Table 1 shows the list of curves and control points of the reference design. The control points can be formed as control polygon. Control polygon acts as a frame to form the curve.

Table 1: List of curve and control points

\begin{tabular}{lllll}
\hline Curve & & \multicolumn{2}{c}{ Control Points } & \\
\hline Curve 1 & $\mathrm{cp} 1=(0.0,0.4)$ & $\mathrm{cp} 2=(0.4,0.8)$ & $\mathrm{cp} 3=(1.4,0.6)$ & $\mathrm{cp} 4=(1.4,0.0)$ \\
Curve 2 & $\mathrm{cp} 4=(1.4,0.0)$ & $\mathrm{cp} 5=(2.0,0.0)$ & $\mathrm{cp} 6=(3.0,0.0)$ & $\mathrm{cp} 7=(3.8,0.0)$ \\
Curve 3 & $\mathrm{cp} 7=(4.8,0.0)$ & $\mathrm{cp} 8=(4.4,0.4)$ & $\mathrm{cp} 9=(4.4,0.8)$ & $\mathrm{cp} 10=(3.8,1.0)$ \\
Curve 4 & $\mathrm{cp} 10=(3.8,1.0)$ & $\mathrm{cp} 11=(4.2,1.2)$ & $\mathrm{cp} 12=(4.4,1.8)$ & $\mathrm{cp} 13=(3.8,2.0)$ \\
Curve 5 & $\mathrm{cp} 13=(3.8,2.0)$ & $\mathrm{cp} 14=(2.4,4.8)$ & $\mathrm{cp} 15=(2.4,6.8)$ & $\mathrm{cp} 16=(3.8,8.0)$ \\
Curve 6 & $\mathrm{cp} 16=(3.8,8.0)$ & $\mathrm{cp} 17=(4.6,8.6)$ & $\mathrm{cp} 18=(4.6,9.4)$ & $\mathrm{cp} 19=(3.8,10.0)$ \\
Curve 7 & $\mathrm{cp} 19=(3.8,10.0)$ & $\mathrm{cp} 20=(4.6,10.4)$ & $\mathrm{cp} 21=(4.6,10.8)$ & $\mathrm{cp} 22=(3.8,11.0)$ \\
Curve 8 & $\mathrm{cp} 22=(3.8,11.0)$ & $\mathrm{cp} 23=(4.6,11.4)$ & $\mathrm{cp} 24=(4.6,11.8)$ & $\mathrm{cp} 25=(4.0,12.6)$ \\
Curve 9 & $\mathrm{cp} 25=(4.0,12.6)$ & $\mathrm{cp} 26=(6.0,14.8)$ & $\mathrm{cp} 27=(4.8,18.0)$ & $\mathrm{cp} 28=(2.8,19.0)$ \\
\hline
\end{tabular}
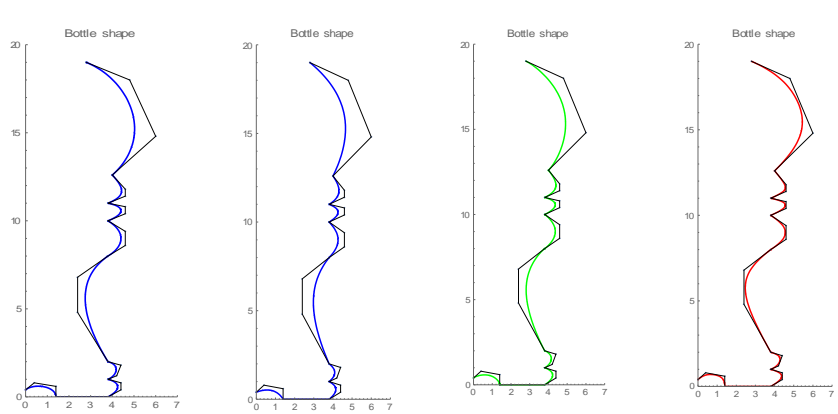

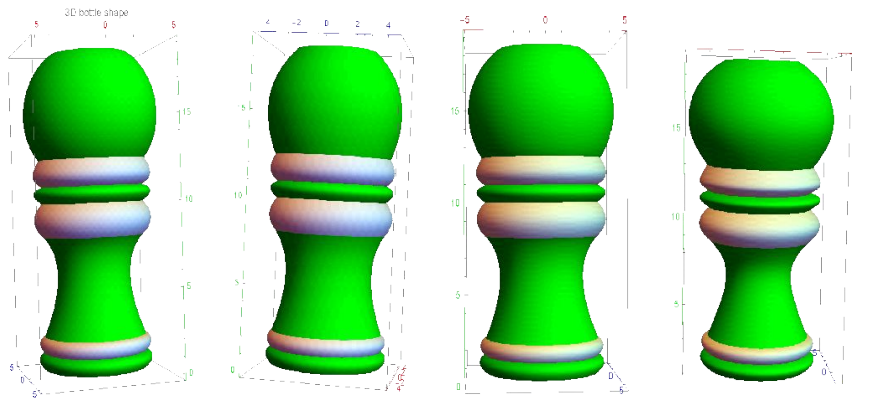

$\lambda=-2$

$\lambda=1$
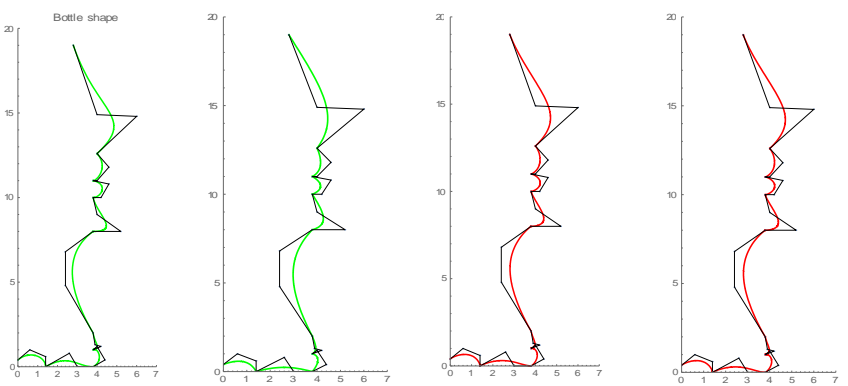

\section{a) Bezier \\ curve}

Figure 5: The 3-dimensional designs

Figure 5 shows the designs of bottles that are generated from the control points defined form reference figure. From the designs, it shows that the Bezier curve can create a design while the trigonometric curve can create more designs by manipulating the shape parameter. The shape parameter has a range from -2 to 1 , but this paper only shows 3 designs based on 3 values of shape parameter which are $-2,-1$ and 1 .

\subsection{Manipulation of the 3-Dimensional Designs}

Based on the properties of the curves, the manipulation of the designs has been made in order to generate new designs from the same of reference figure. In this paper, it shows how the properties in this curve can generate new designs easily and can also create a unique design. The manipulation was done by manipulating the control points and also the shape parameter. The Bezier curve just involved with manipulation of control points but for Trigonometric curve, the manipulation was done for both. Here, it shows the properties of Trigonometric curve has more advantages as compared to the Bezier curve and furthermore, more designs can be generated.

Table 2: The manipulation of the control points

\begin{tabular}{cc}
\hline Control Point Original & Manipulated Control Point \\
\hline $\mathrm{cp} 2=(0.4,0.8)$ & $\mathrm{cp} 2=(0.6,1.0)$ \\
$\mathrm{cp} 5=(2.0,0.0)$ & $\mathrm{cp} 5=(2 \cdot 6,0.8)$ \\
$\mathrm{cp} 9=(4.4,0.8)$ & $\mathrm{cp} 9=(4.0,1.2)$ \\
$\mathrm{cp} 12=(4.4,1.8)$ & $\mathrm{cp} 12=(3.9,1.3)$ \\
$\mathrm{cp} 17=(4.6,8.6)$ & $\mathrm{cp} 17=(5 \cdot 2,8.0)$ \\
$\mathrm{cp} 18=(4.6,9.4)$ & $\mathrm{cp} 18=(4.0,9.0)$ \\
$\mathrm{cp} 20=(4.6,10.4)$ & $\mathrm{cp} 20=(4.2,10.0)$ \\
$\mathrm{cp} 23=(4.6,11.4)$ & $\mathrm{cp} 23=(4.0,11.0)$ \\
$\mathrm{cp} 27=(4.8,18.0)$ & $\mathrm{cp} 27=(4.0,14.9)$
\end{tabular}
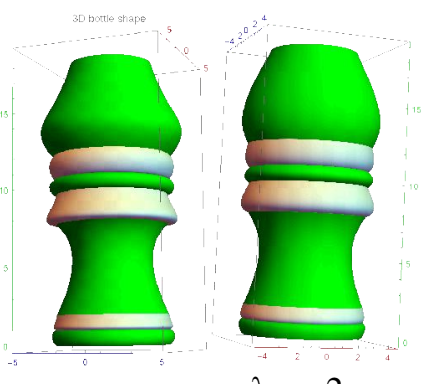

$\lambda=-2$

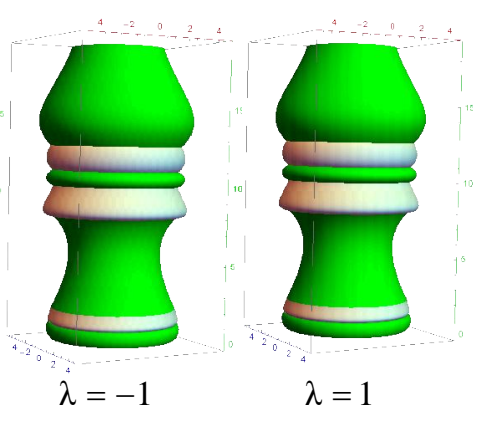

b) Trigonometric curve a) Bezier curve

Figure 6: The 3-dimensional designs based on manipulation of control points

Table 2 show the values of control points that were manipulated to generate the new designs. All the designs are shown in Figure 6. All the designs changed when the control points changed. It is because the control points affect the shape of control polygon. Besides that, for trigonometric curve, the value shape parameter also can generate more designs as compared to Bezier curve.
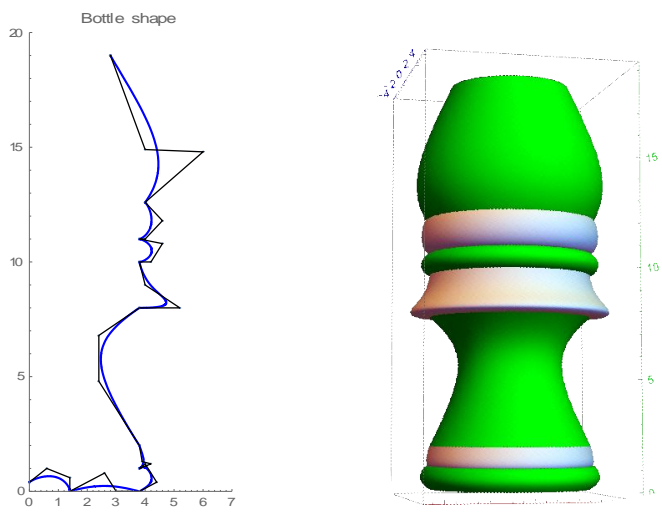

Figure 7: The 3-dimensional designs of Trigonometric curve based on manipulation of control points and shape parameter

Figure 7 shows the design that was generated from Trigonometric curve with different values of shape parameter for each curve. Since the curves are combined with different curves, each curve can be used with the different value of shape parameter. The values of shape parameter use are $-2,-1$ and 1 . However, the designer can use others values as long as they are within the range. Here, different designs also can be produced and it requires simple steps to generate the unique designs. This property can help the designer to create more and variety designs easily by manipulating the value of shape 
parameter and control points. It also can reduce the cost of time and also reduce the complexity in designing the 3-dimensional objects.

\section{CONCLUSION}

In order to generate the best design in designing a design, the shape of design is the most important aspect to be considered and should be emphasized. This is also part of a marketing strategy. The design can also be conveyed with color, fonts, and textures, thus made the design stand out from other designs. Furthermore, nowadays, with the advancement of technology, there are some methods and technology applications that can create digital design.

The methods that were used in this paper is Cubic Bezier Curve and Cubic Trigonometry with a Shape Parameter. Both methods act as an alternative method besides the common methods that are used nowadays. The curve of Cubic Bezier Curve and Cubic Trigonometry with a Shape Parameter are used in generating the 2-Dimensional design. Bezier Curve is a parametric curve that has the polynomial capacity of the parameter $t$. The level polynomial relies upon the amount of focuses used to characterize the curve. Each control point will impact the heading of the curve by dragging it towards itself, and mostly grounded when the curve is the closest to the control points. Cubic Trigonometry with a Shape Parameter is similar to Bezier curve, but with a shape parameter. The curve can be adjusted not only with control points but also can be made by using changing the value of the shape parameter, $\lambda$. These are the advantages because this method is easier to create curve and design. From both methods, 2-Dimensional design can be created. Then, 3-Dimensional symmetrical objects can be generated using the Sweep Surface Rotation method. A swept surface is created in creating the curve and moving lengthways with a new curve, though optionally rotating and scaling, to create a surface area. To create a surface path, the curve is rotated about a y-axis.

As a conclusion, the best method used is Cubic Trigonometry with a Shape Parameter because it is easier to manipulate the curve with a shape parameter as compared to Bezier Curve. Furthermore, the application also can be used other methods such as Spline, Rational Curve and also can be applied with other different sweeping methods in generating 3-dimensional designs.

\section{ACKNOWLEDGEMENT}

The authors would like to acknowledge UiTM Terengganu Branch Kuala Terengganu Campus for its support. All the objects were generated using Wolfram Mathematica software.

\section{REFERENCES}

1. Dube, M., \& Yadav, B. The quantic trigonometric Bezier curve with single shape parameter. International Journal scientific and Research publications, 2014, 4(3), 2250-3153.

2. Farin, G. Class a Bézier curves. Computer Aided Geometric Design. 2006. 23(7), 573-581. https://doi.org/10.1016/j.cagd.2006.03.004

3. Han, X. A., Ma, Y., \& Huang, X. The cubic trigonometric Bézier curve with two shape parameters. Applied Mathematics Letters, 2009, 22(2), 226-231.

https://doi.org/10.1016/j.aml.2008.03.015

4. Han, X. A., Huang, X., \& Ma, Y. Shape analysis of cubic trigonometric Bézier curves a shape parameter. Applied Mathematics and Computation, 2010, 217(6),2527-2533. https://doi.org/10.1016/j.amc.2010.07.065

5. Johnstone, J. K., \& Williams, J. P. A rational model of the surface swept by a curve. In Computer Graphics Forum (Vol. 14, No. 3, pp. 77-88). Edinburgh, UK: Blackwell Science Ltd, August 1995.

6. Morin, G. Analytic functions in computer-aided geometric design, $\mathrm{Ph}$.D. dissertation, 2002.

7. Salamon, S. M. Differential geometry of quaternionic manifolds. In Annales scientifiques de l'Ecole normale supérieure, 1986, vol. 19, no. 1, pp. 31-55.

8. Salomon, D. Curves and surfaces for computer graphics. Springer Science \& Business Media, 2007.

9. Salomon, D. Curves and Surfaces for Computer Graphics. California: Springer.2006

10. Sukri, N. M., Razali, N. K., Norddin, N. I., Al Din, S. M. N., Azaman, W. A. W., \& Rizman, Z. I. Development of 3D cartoon by using B-spline and sweep surface method. International Journal of Advanced and Sciences, 2017, 4(12), 158-161.

11. Yan, L., Liang, J., \& Wu, G. Two Kinds of Trigonometric Spline Curves with Shape Parameter. In 2009 International Conference on Environmental Science and Information Application Technology (Vol. 1, pp. 549-552). IEEE, July 2009.

12. Sharma, R. Cubic Trigonometric Bézier Curve with Shape Parameter. International Journal of Innovative Research in Computer and Communication Engineering. 2016. 4(4). 7718-7723.

13. Rocchi, Benedetto, Stefani \& Gianluca. Consumers' perception of wine packaging: A case study. 2016. 18. 33-44. 